\title{
Obtaining More Karatsuba-Like Formulae over the Binary Field
}

\author{
Haining Fan, Ming Gu, Jiaguang Sun and Kwok-Yan Lam
}

\begin{abstract}
The aim of this paper is to find more Karatsuba-like formulae for a fixed set of moduli polynomials in $G F(2)[x]$. To this end, a theoretical framework is established. We first generalize the division algorithm, and then present a generalized definition of the remainder of integer division. Finally, a previously generalized Chinese remainder theorem is used to achieve our initial goal. As a by-product of the generalized remainder of integer division, we rediscover Montgomery's $N$-residue and present a systematic interpretation of definitions of Montgomery's multiplication and addition operations.
\end{abstract}

\section{Index Terms}

Karatsuba algorithm, polynomial multiplication, Chinese remainder theorem, Montgomery algorithm, finite field.

\section{INTRODUCTION}

Efficient $G F\left(2^{n}\right)$ multiplication operation is important in cryptosystems. The main advantage of subquadratic multipliers is that their low asymptotic space complexities make it possible to implement VLSI multipliers for large values of $n$. The Karatsuba algorithm, which was invented by Karatsuba in 1960 [1], provides a practical solution for subquadratic $G F\left(2^{n}\right)$ multipliers [2]. Because time and space complexities of these multipliers depend on low-degree Karatsuba-like formulae, much effort has been devoted to obtain Karatsuba-like formulae with low multiplication complexity. Using the Chinese remainder theorem (CRT), Lempel, Seroussi and Winograd obtained a quasi-linear upper bound of the multiplicative complexity of multiplying

Haining Fan, Ming Gu, Jiaguang Sun and Kwok-Yan Lam are with the School of Software, Tsinghua University, Beijing, China. E-mails: $\{$ fhn, guming, sunjg, lamky $\} @$ tsinghua.edu.cn 
two polynomials over finite fields [3]. Weimerskirch and Paar generalized the Karatsuba algorithm and showed how to use it with the least number of operations [4]. Based on an exhaustive search method, Montgomery presented Karatsuba-like formulae which multiply two polynomials of degree at most 4, 5, or 6 in $G F(2)[x]$ [5]. He also obtained new upper bounds on the multiplication complexity of $n$-term (degree $n-1)$ polynomials for some small $n$. Recently, some bounds in [5] were improved by Fan and Hasan [6], Cenk and Özbudak [7], Oseledets [8] and Cenk, Koç and Özbudak [9].

Apart from Weimerskirch and Paar's method, the above methods can be classified into two categories: the exhaustive search method [5] [8] and the CRT-based method [3] [6] [7] [8] [9]. The exhaustive search method can find all $n$-term Karatsuba-like formulae for a fixed value of $n$, but its drawback is obvious, namely, it can only be used for small values of $n$. The CRT-based method is suitable for both small and large values of $n$, but only one $n$-term Karatsuba-like formula can be derived once the set of moduli polynomials is chosen.

The purpose of this paper is to find more Karatsuba-like formulae for a fixed set of moduli polynomials in $G F(2)[x]$. To this end, a theoretical framework is established. We first generalize the division algorithm, and then present a generalized definition of the remainder of integer division. As a by-product of these generalizations, we find that the residue class determined by this generalized remainder turns out to be Montgomery's $N$-residue [11]; and furthermore, we present a systematic interpretation of definitions of Montgomery's multiplication and addition operations. Finally, a previously generalized CRT is used to achieve our initial goal.

The remainder of this article is organized as follows: We present the generalized division algorithm in Section II. After presenting two examples in Section III, we summarize a method to obtain more Karatsuba-like formulae. Finally, concluding remarks are made in Section IV.

\section{A Generalization of the Division Algorithm}

\section{A. A Generalization of the Division Algorithm}

The integer division algorithm is the basis of the congruence theory.

Theorem 1 (The division algorithm): $\forall 0<m, a \in \mathbb{Z}$, there exist unique integers $q^{\prime}$ and $r^{\prime}$ with $0 \leq r^{\prime}<m$ such that $a=m \cdot q^{\prime}+r^{\prime}$.

Based on Theorem 1, we have the classical definition of the remainder of a modulo $m$, i.e., 
Definition 1: $\forall 0<m, a \in \mathbb{Z}$, the remainder of $a$ modulo $m$ is defined as $a \bmod m:=r^{\prime}=$ $a-m q^{\prime}$, where $r^{\prime}$ and $q^{\prime}$ are unique integers determined by Theorem 1 .

More precisely, $r^{\prime}$ in Theorem 1 is called the least non-negative remainder. In the following, we will use $\langle a\rangle_{m}$ to denote $a \bmod m$. Before we present the proposed generalization of Theorem 1, we introduce another generalization of the division algorithm.

Theorem 2 (The 1st generalization of the division algorithm): $\forall 0<m, a, d \in \mathbb{Z}$, there exist unique integers $q^{\prime}$ and $r^{\prime}$ with $d \leq r^{\prime}<m+d$ such that $a=m q^{\prime}+r^{\prime}$.

Especially, if $d=-\left\lfloor\frac{m}{2}\right\rfloor$ then $-\left\lfloor\frac{m}{2}\right\rfloor \leq r^{\prime}<m-\left\lfloor\frac{m}{2}\right\rfloor$. In this case, $r^{\prime}$ is called the least absolute remainder. As an application of this generalization, the original Euclidean algorithm for integers can be slightly speeded up [10, Exercise 3.13 and 3.30].

Let $\mathbb{Z}_{m}^{*}=\left\{i \mid i \in \mathbb{Z}_{m}\right.$ and $\left.\operatorname{gcd}(i, m)=1\right\}$ be the multiplicative group of $\mathbb{Z}_{m}$ and "." denote the multiplication operation in $\mathbb{Z}$. The second generalization of the division algorithm is as follows.

Proposition 3 (The 2nd generalization of the division algorithm): $\forall 0<m, a \in \mathbb{Z}$. Let $R^{-1} \in$ $\mathbb{Z}_{m}^{*}$ be the multiplicative inverse of $R \in \mathbb{Z}_{m}^{*}$. Then there exist unique integers $q$ and $r$ with $0 \leq r<m$ such that $a=m \cdot q+R^{-1} \cdot r$.

Proof:

$\because R^{-1}$ is the multiplicative inverse of $R$ in $\mathbb{Z}_{m}^{*}$,

$\therefore \exists u \in \mathbb{Z}$ such that $1=u m+R R^{-1}$.

$\therefore a=a u m+a R R^{-1}$.

By the division algorithm, there exist unique integers $q^{\prime \prime}$ and $r^{\prime \prime}$ such that $a R=m q^{\prime \prime}+r^{\prime \prime}$, where $0 \leq r^{\prime \prime}=\langle a R\rangle_{m}<m$. Therefore, $a=a u m+(a R) R^{-1}$ can be rewritten as

$$
\begin{aligned}
a & =a u m+\left(m q^{\prime \prime}+r^{\prime \prime}\right) R^{-1} \\
& =a u m+m q^{\prime \prime} R^{-1}+R^{-1} r^{\prime \prime} \\
& =\left(a u+q^{\prime \prime} R^{-1}\right) m+R^{-1} r^{\prime \prime}
\end{aligned}
$$

$\therefore$ There exist integers $q=\left(u a+q^{\prime \prime} R^{-1}\right)$ and $r=r^{\prime \prime}=\langle a R\rangle_{m}$ with $0 \leq r<m$ such that $a=m q+R^{-1} r$.

To prove the uniqueness, we assume, on the contrary, that there exist $q_{1}, q_{2}$, and $0 \leq r_{1}, r_{2}<m$ such that $a=m \cdot q_{1}+R^{-1} \cdot r_{1}=m \cdot q_{2}+R^{-1} \cdot r_{2}$.

If $r_{1}=r_{2}$ then it is easy to prove that $q_{1}=q_{2}$. 
For the case $r_{1} \neq r_{2}$, since $\left(m, R^{-1}\right)=1$ and $m$ divides $0=a-a=m\left(q_{1}-q_{2}\right)+R^{-1} \cdot\left(r_{1}-r_{2}\right)$, we have $r_{1}=r_{2}$. This is a contradiction.

Obviously, Proposition 3 becomes Theorem 1 when $R=R^{-1}=1$.

Because the classical definition of the remainder of $a$ modulo $m$, i.e., $\langle a\rangle_{m}$ in Definition 1 , is based on the classical division algorithm Theorem 1, and we have just generalized Theorem 1 to Proposition 3, the unique integer $r=\langle a R\rangle_{m}$ appeared in the proof of Proposition 3 can be naturally viewed as a generalization of $\langle a\rangle_{m}$, i.e.,

Definition 2 (A generalized remainder of a modulo $m$ ): $\forall 0<m, a \in \mathbb{Z}$ and $R \in \mathbb{Z}_{m}^{*}$. The generalized remainder of $a$ modulo $m$ w.r.t. $R$ is defined as $\langle a\rangle_{(m, R)}:=\langle a \cdot R\rangle_{m}$.

The reader may be familiar with $\langle a R\rangle_{m}$. In fact, it corresponds to the $N$-residue of $a$ defined by Montgomery in [11]. Montgomery's representation involves only one parameter $R$. Using the generalized division algorithm, we can readily deal with two or more $R$ 's. The following equation is such an example, and it will be used in the next section.

$$
\langle a b\rangle_{\left(m, R_{c}\right)}=\left\langle a R_{a} \cdot b R_{b} \cdot \frac{R_{c}}{R_{a} R_{b}}\right\rangle_{m}=\left\langle\langle a\rangle_{\left(m, R_{a}\right)} \cdot\langle b\rangle_{\left(m, R_{b}\right)} \cdot \frac{R_{c}}{R_{a} R_{b}}\right\rangle_{m} .
$$

B. A Systematic Interpretation of Definitions of Montgomery's Multiplication and Addition Operations

Let $a=m \cdot q_{a}+R^{-1} \cdot r_{a}$ and $b=m \cdot q_{b}+R^{-1} \cdot r_{b}$ be two positive integers, whose $N$ residues correspond to $r_{a}=\langle a\rangle_{(m, R)}=\langle a \cdot R\rangle_{m}$ and $r_{b}=\langle b\rangle_{(m, R)}=\langle b \cdot R\rangle_{m}$ respectively. In Montgomery's representation, the addition operation " $\oplus$ ", i.e., $r_{a} \oplus r_{b}:=\left\langle r_{a}+r_{b}\right\rangle_{m}$, is defined the same as that in $\mathbb{Z}_{m}$. But the definition of the multiplication operation " $\otimes$ " is different, which is defined as $r_{a} \otimes r_{b}:=\left\langle r_{a} \cdot r_{b} \cdot R^{-1}\right\rangle_{m}$. The reason that operation " $\otimes$ " is defined in this way, not other expressions, can be traced back to the $N$-residue of $a \cdot b$, which is uniquely determined by the generalized division algorithm. Or, more precisely, expanding $a \cdot b=$ $\left(m \cdot q_{a}+R^{-1} \cdot r_{a}\right)\left(m \cdot q_{b}+R^{-1} \cdot r_{b}\right)$ as

$$
a \cdot b=m\left(m q_{a} q_{b}+q_{a} R^{-1} r_{b}+q_{b} R^{-1} r_{a}\right)+R^{-1}\left(R^{-1} \cdot r_{a} \cdot r_{b}\right)
$$

and expressing $\left(R^{-1} \cdot r_{a} \cdot r_{b}\right)$ as $R^{-1} \cdot r_{a} \cdot r_{b}=m\left\lfloor\frac{R^{-1} \cdot r_{a} \cdot r_{b}}{m}\right\rfloor+\left\langle R^{-1} \cdot r_{a} \cdot r_{b}\right\rangle_{m}$ by the division algorithm, we have

$$
a \cdot b=m\left(m q_{a} q_{b}+q_{a} R^{-1} r_{b}+q_{b} R^{-1} r_{a}+R^{-1}\left\lfloor\frac{R^{-1} \cdot r_{a} \cdot r_{b}}{m}\right\rfloor\right)+R^{-1}\left[\left\langle R^{-1} \cdot r_{a} \cdot r_{b}\right\rangle_{m}\right] .
$$


By Proposition 3 and Definition 2, the integer $\left\langle R^{-1} \cdot r_{a} \cdot r_{b}\right\rangle_{m}=\langle(a \cdot b) R\rangle_{m}$ in the square brackets just corresponds to the $N$-residue of $a \cdot b$.

The definition of Montgomery's addition operation of $N$-residues can also be interpreted similarly: expressing $a+b$ by the generalized division algorithm as

$$
\begin{aligned}
a+b & =\left(m \cdot q_{a}+R^{-1} \cdot r_{a}\right)+\left(m \cdot q_{b}+R^{-1} \cdot r_{b}\right) \\
& =m\left(q_{a}+q_{b}\right)+R^{-1}\left(r_{a}+r_{b}\right) \\
& =m\left(q_{a}+q_{b}+R^{-1}\left\lfloor\frac{r_{a}+r_{b}}{m}\right\rfloor\right)+R^{-1}\left\langle r_{a}+r_{b}\right\rangle_{m}
\end{aligned}
$$

the integer $\left\langle r_{a}+r_{b}\right\rangle_{m}$ corresponds to Montgomery's summation of two $N$-residues $r_{a}$ and $r_{b}$, i.e., $r_{a} \oplus r_{b}$.

\section{A Generalization of the CRT}

The following is an integer version of the CRT.

Theorem $4(C R T)$ : Let $t>1, m_{1}, m_{2}, \cdots, m_{t}$ be pairwisely coprime positive integers, $M=$ $\prod_{i=1}^{t} m_{i}$ and $M_{i}=\frac{M}{m_{i}}$. Then the unique solution $y$ modulo $M$ to the system of linear congruences $\langle y\rangle_{m_{i}}=y_{i}^{\prime}$ is

$$
y=\left\langle\sum_{i=1}^{t} y_{i}^{\prime} \cdot M_{i} \cdot\left\langle M_{i}^{-1}\right\rangle_{m_{i}}\right\rangle_{M},
$$

where $\left\langle M_{i}^{-1}\right\rangle_{m_{i}}$ is the multiplicative inverse of $M_{i}$ in $\mathbb{Z}_{m_{i}}^{*}$ and $1 \leq i \leq t$.

In the above subsection, we have presented a generalized definition of the remainder of integer division. Therefore, it is natural to seek the solution to the system of the generalized linear congruences $\langle y\rangle_{\left(m_{i}, R_{i}\right)}=y_{i}$. This consideration leads to a rediscovery of the following generalized CRT [12]:

Theorem 5 (A generalized CRT): Let $t>1, m_{1}, m_{2}, \cdots, m_{t}$ be pairwisely coprime positive integers, $M=\prod_{i=1}^{t} m_{i}, M_{i}=\frac{M}{m_{i}}$ and $R_{i} \in \mathbb{Z}_{m_{i}}^{*}$. Then the unique solution $y$ modulo $M$ to the system of generalized linear congruences $\langle y\rangle_{\left(m_{i}, R_{i}\right)}=y_{i}$ is

$$
y=\left\langle\sum_{i=1}^{t} y_{i} \cdot M_{i} \cdot\left\langle\left\langle M_{i}^{-1}\right\rangle_{m_{i}} \cdot\left\langle R_{i}^{-1}\right\rangle_{m_{i}}\right\rangle_{m_{i}}\right\rangle_{M},
$$

where $\left\langle M_{i}^{-1}\right\rangle_{m_{i}}$ and $\left\langle R_{i}^{-1}\right\rangle_{m_{i}}$ are multiplicative inverses of $M_{i}$ and $R_{i}$ in $\mathbb{Z}_{m_{i}}^{*}$ respectively and $1 \leq i \leq t$ 
The correctness of this theorem is clear since the system of linear congruences $\langle y\rangle_{\left(m_{i}, R_{i}\right)}=$ $\left\langle y \cdot R_{i}\right\rangle_{m_{i}}=y_{i}$ is equivalent to the system of linear congruences $\langle y\rangle_{m_{i}}=\left\langle y_{i} \cdot R_{i}^{-1}\right\rangle_{m_{i}}$, which has the solution (3) by (2).

Until now, we have focussed only on the ring $\mathbb{Z}_{m}$. In fact, these results can be transferred to the polynomial ring $F[x]$ without essential modification, where $F$ is a field. For simplicity, we do not rewrite them here.

\section{Obtaining More Karatsuba-Like Formulae in $G F(2)[x]$}

We now use the above results to obtain more Karatsuba-like formulae for a fixed set of moduli polynomials in $G F(2)[x]$. Two examples are presented first to illustrate the main idea.

\section{A. 3-term Karatsuba-like Formulae}

This example provides all 3-term Karatsuba-like formulae that can be derived from the generalized CRT Theorem 5. These formulae compute $C=\sum_{i=0}^{4} c_{i} x^{i}=A B=\left(a_{2} x^{2}+a_{1} x+\right.$ $\left.a_{0}\right)\left(b_{2} x^{2}+b_{1} x+b_{0}\right)$ in $G F(2)[x]$ using 6 multiplications.

For the purpose of comparison, we first present the formula derived from the conventional CRT. The moduli polynomials used in this example are $f_{\infty}=x-\infty, f_{0}=x, f_{1}=x+1$ and $f_{2}=x^{2}+x+1$. We will not present the detailed procedure to construct the whole Karatsuba-like formula. Instead, we present only the computation procedure of the term $\left\langle y_{2}^{\prime} \cdot M_{2} \cdot\left\langle M_{2}^{-1}\right\rangle_{f_{2}}\right\rangle_{M}$ appeared in the conventional CRT, which will be called the product term in the following.

For moduli polynomial $f_{2}=x^{2}+x+1$. We first compute parameters $M=f_{0} \cdot f_{1} \cdot f_{2}=x^{4}+x$, $M_{2}=\frac{M}{f_{2}}=x^{2}+x$ and $\left\langle M_{2}^{-1}\right\rangle_{f_{2}}=1$. Then we compute the product term as follows:

$$
\begin{aligned}
& \left\langle\langle A B\rangle_{f_{2}} \cdot M_{2} \cdot\left\langle M_{2}^{-1}\right\rangle_{f_{2}}\right\rangle_{M} \\
= & \left\langle\left\langle\langle A\rangle_{f_{2}} \cdot\langle B\rangle_{f_{2}}\right\rangle_{f_{2}} \cdot M_{2} \cdot\left\langle M_{2}^{-1}\right\rangle_{f_{2}}\right\rangle_{M} \\
= & \left\langle\left\langle\left[\left(a_{1}+a_{2}\right) x+\left(a_{0}+a_{2}\right)\right] \cdot\left[\left(b_{1}+b_{2}\right) x+\left(b_{0}+b_{2}\right)\right]\right\rangle_{f_{2}} \cdot\left(x^{2}+x\right) \cdot 1\right\rangle_{M} \\
= & \left\langle\left\langle m_{4} x^{2}+\left(m_{3}+m_{4}+m_{5}\right) x+m_{3}\right\rangle_{f_{2}} \cdot\left(x^{2}+x\right)\right\rangle_{M} \\
= & {\left[\left(m_{3}+m_{5}\right) x+\left(m_{3}+m_{4}\right)\right] \cdot\left(x^{2}+x\right) } \\
= & \left(m_{3}+m_{5}\right) x^{3}+\left(m_{4}+m_{5}\right) x^{2}+\left(m_{3}+m_{4}\right) x,
\end{aligned}
$$

where $m_{3}=\left(a_{0}+a_{2}\right)\left(b_{0}+b_{2}\right), m_{4}=\left(a_{1}+a_{2}\right)\left(b_{1}+b_{2}\right)$ and $m_{5}=\left(a_{0}+a_{1}\right)\left(b_{0}+b_{1}\right)$. 
After getting the two product terms corresponding to two other moduli polynomials $f_{0}=x$ and $f_{1}=x+1$, we can obtain the CRT-based 3-term Karatsuba-like formula using the construction multiplication modulo $(x-\infty)^{w}\left[6\right.$, Lemma 2]. The formula is listed in table I as $\mathcal{F}_{1}$.

TABLE I

All 3-TERM KARATSUBA-LIKE FORMULAE OBTAINED FROM THEOREM 5

\begin{tabular}{|c|c|c|c|}
\hline No. & $\left(R_{A}, R_{B}\right)$ & $c_{i}$ 's & The six multiplications \\
\hline $\mathcal{F}_{1}$ & $\begin{array}{c}(1,1), \\
(x, x), \\
(x+1, x+1)\end{array}$ & $\begin{array}{l}c_{0}=m_{0} \\
c_{1}=m_{1}+m_{2}+m_{3}+m_{4} \\
c_{2}=m_{1}+m_{4}+m_{5} \\
c_{3}=m_{0}+m_{1}+m_{3}+m_{5} \\
c_{4}=m_{2}\end{array}$ & $\begin{array}{l}m_{0}=a_{0} b_{0} \\
m_{1}=\left(a_{0}+a_{1}+a_{2}\right)\left(b_{0}+b_{1}+b_{2}\right) \\
m_{2}=a_{2} b_{2} \\
m_{3}=\left(a_{0}+a_{2}\right)\left(b_{0}+b_{2}\right) \\
m_{4}=\left(a_{1}+a_{2}\right)\left(b_{1}+b_{2}\right) \\
m_{5}=\left(a_{0}+a_{1}\right)\left(b_{0}+b_{1}\right)\end{array}$ \\
\hline $\mathcal{F}_{2}$ & $\begin{array}{c}(x, 1), \\
(1, x+1), \\
(x+1, x)\end{array}$ & $\begin{array}{l}c_{0}=m_{0} \\
c_{1}=m_{1}+m_{2}+m_{4}+m_{5} \\
c_{2}=m_{1}+m_{3}+m_{5} \\
c_{3}=m_{0}+m_{1}+m_{3}+m_{4} \\
c_{4}=m_{2},\end{array}$ & $\begin{array}{l}m_{0}=a_{0} b_{0} \\
m_{1}=\left(a_{0}+a_{1}+a_{2}\right)\left(b_{0}+b_{1}+b_{2}\right) \\
m_{2}=a_{2} b_{2} \\
m_{3}=\left(a_{1}+a_{2}\right)\left(b_{0}+b_{2}\right) \\
m_{4}=\left(a_{0}+a_{1}\right)\left(b_{1}+b_{2}\right) \\
m_{5}=\left(a_{0}+a_{2}\right)\left(b_{0}+b_{1}\right)\end{array}$ \\
\hline $\mathcal{F}_{3}$ & $\begin{array}{c}(1, x), \\
(x+1,1), \\
(x, x+1)\end{array}$ & $\begin{array}{l}c_{0}=m_{0} \\
c_{1}=m_{1}+m_{2}+m_{4}+m_{5} \\
c_{2}=m_{1}+m_{3}+m_{5} \\
c_{3}=m_{0}+m_{1}+m_{3}+m_{4} \\
c_{4}=m_{2}\end{array}$ & $\begin{array}{l}m_{0}=a_{0} b_{0} \\
m_{1}=\left(a_{0}+a_{1}+a_{2}\right)\left(b_{0}+b_{1}+b_{2}\right) \\
m_{2}=a_{2} b_{2} \\
m_{3}=\left(a_{0}+a_{2}\right)\left(b_{1}+b_{2}\right) \\
m_{4}=\left(a_{1}+a_{2}\right)\left(b_{0}+b_{1}\right) \\
m_{5}=\left(a_{0}+a_{1}\right)\left(b_{0}+b_{2}\right)\end{array}$ \\
\hline
\end{tabular}

Now we present the new formula derived from the generalized CRT Theorem 5. We need to generalize the two remainders $\langle A\rangle_{f_{2}}=\langle A\rangle_{\left(f_{2}, 1\right)}$ and $\langle B\rangle_{f_{2}}=\langle B\rangle_{\left(f_{2}, 1\right)}$ appeared in (4) to $\langle A\rangle_{\left(f_{2}, R_{A}\right)}$ and $\langle B\rangle_{\left(f_{2}, R_{B}\right)}$, where $R_{A}$ and $R_{B}$ belong to the multiplicative group $G F(2)[x] /\left(f_{2}\right)^{*}=$ $\{1, x, x+1\}$. Setting $\left(R_{A}, R_{B}\right)=(x, 1)$, we have $\langle A\rangle_{\left(f_{2}, R_{A}\right)}=\langle A \cdot x\rangle_{f_{2}}=\left(a_{0}+a_{1}\right) x+\left(a_{1}+a_{2}\right)$ 
and $\left\langle R_{A}^{-1}\right\rangle_{f_{2}}=x+1$. Then we obtain the following product term by (1).

$$
\begin{aligned}
& \left\langle\langle A B\rangle_{f_{2}} \cdot M_{2} \cdot\left\langle M_{2}^{-1}\right\rangle_{f_{2}}\right\rangle_{M} \\
= & \left\langle\left\langle\left(A \cdot R_{A} \cdot R_{A}^{-1}\right) \cdot\left(B \cdot R_{B} \cdot R_{B}^{-1}\right)\right\rangle_{f_{2}} \cdot M_{2} \cdot\left\langle M_{2}^{-1}\right\rangle_{f_{2}}\right\rangle_{M} \\
= & \left\langle\left\langle\langle A\rangle_{\left(f_{2}, R_{A}\right)} \cdot\langle B\rangle_{\left(f_{2}, R_{B}\right)}\right\rangle_{f_{2}} \cdot M_{2} \cdot\left\langle M_{2}^{-1} \cdot R_{A}^{-1} \cdot R_{B}^{-1}\right\rangle_{f_{2}}\right\rangle_{M} \\
= & \left\langle\left\langle\langle A\rangle_{\left(f_{2}, x\right)} \cdot\langle B\rangle_{f_{2}}\right\rangle_{f_{2}} \cdot\left(x^{2}+x\right) \cdot(x+1)\right\rangle_{M} \\
= & \left\langle\left\langle\left[\left(a_{0}+a_{1}\right) x+\left(a_{1}+a_{2}\right)\right] \cdot\left[\left(b_{1}+b_{2}\right) x+\left(b_{0}+b_{2}\right)\right]\right\rangle_{f_{2}} \cdot\left(x^{3}+x\right)\right\rangle_{M} \\
= & \left\langle\left\langle m_{4} x^{2}+\left(m_{3}+m_{4}+m_{5}\right) x+m_{3}\right\rangle_{f_{2}} \cdot\left(x^{3}+x\right)\right\rangle_{M} \\
= & \left\langle\left[\left(m_{3}+m_{5}\right) x+\left(m_{3}+m_{4}\right)\right] \cdot\left(x^{3}+x\right)\right\rangle_{x^{4}+x} \\
= & \left(m_{3}+m_{4}\right) x^{3}+\left(m_{3}+m_{5}\right) x^{2}+\left(m_{4}+m_{5}\right) x
\end{aligned}
$$

where $m_{3}=\left(a_{1}+a_{2}\right)\left(b_{0}+b_{2}\right), m_{4}=\left(a_{0}+a_{1}\right)\left(b_{1}+b_{2}\right)$ and $m_{5}=\left(a_{0}+a_{2}\right)\left(b_{0}+b_{1}\right)$.

The remaining steps to construct the new 3-term Karatsuba-like formula are the same as those in the conventional CRT, and we list this new formula $\mathcal{F}_{2}$ in the middle of table I.

It is clear that the CRT-based formula $\mathcal{F}_{1}$ is symmetrical, namely, it does not change if we exchange " $a$ " and " $b$ " in $m_{i}$ 's. But if we exchange " $a$ " and " $b$ " in the new formula $\mathcal{F}_{2}$, we will obtain a brand new formula $\mathcal{F}_{3}$, which can be obtained by setting $\left(R_{A}, R_{B}\right)=(1, x)$. Therefore, formula $\mathcal{F}_{2}$ (or $\mathcal{F}_{3}$ ) is not symmetrical from this point of view.

Since there are 3 elements in $G F(2)[x] /\left(f_{2}\right)^{*}=\{1, x, x+1\}$, we have 9 different combinations of pair $\left(R_{A}, R_{B}\right)$. For each of these pairs, we can obtain one 3-term Karatsuba-like formula. But some of them are the same. For example, the CRT-based formula $\mathcal{F}_{1}$, which is derived by setting $\left(R_{A}, R_{B}\right)=(1,1)$, can also be obtained by setting $\left(R_{A}, R_{B}\right)=(x, x)$ or $\left(R_{A}, R_{B}\right)=$ $(x+1, x+1)$. In table $\mathrm{I}$, all three distinct formulae are listed. Here we note that $f_{2}$ is the only moduli polynomial that the generalized CRT can be applied to because there is only one element, i.e., 1, in either $G F(2)[x] /\left(f_{0}\right)^{*}$ or $G F(2)[x] /\left(f_{1}\right)^{*}$.

\section{B. Another 9-term Karatsuba-like Formula}

A 9-term CRT-based Karatsuba-like formula, which computes $C=\sum_{i=0}^{16} c_{i} x^{i}=A \cdot B=$ $\sum_{i=0}^{8} a_{i} x^{i} \cdot \sum_{i=0}^{8} b_{i} x^{i}$ in $G F(2)[x]$, was given in [7]. They selected the moduli polynomials $(x-\infty)^{3}, f_{11}^{3}=x^{3}, f_{12}^{3}=(x+1)^{3}, f_{21}=x^{2}+x+1, f_{31}=x^{3}+x+1$ and $f_{32}=x^{3}+x^{2}+1$. 
In the following, we will also use these moduli polynomials and derive a new Karatsuba-like formula by generalizing product terms corresponding to moduli polynomials $f_{31}$ and $f_{32}$.

For moduli polynomial $f_{31}$, we select $R_{A}=R_{B}=x$ and compute $\langle A\rangle_{\left(f_{31}, R_{A}\right)}=\langle A \cdot x\rangle_{f_{31}}$ and $\langle B\rangle_{\left(f_{31}, R_{B}\right)}=\langle B \cdot x\rangle_{f_{31}}$ first. Then we compute its product term as follows.

$$
\begin{aligned}
& \left\langle\langle A B\rangle_{f_{31}} \cdot M_{31} \cdot\left\langle\frac{1}{M_{31}}\right\rangle_{f_{31}}\right\rangle_{M} \\
= & \left\langle\left\langle\langle A\rangle_{\left(f_{31}, R_{A}\right)} \cdot\langle B\rangle_{\left(f_{31}, R_{B}\right)}\right\rangle_{f_{31}} \cdot M_{31} \cdot\left\langle\frac{1}{M_{31}} \cdot \frac{1}{R_{A} \cdot R_{B}}\right\rangle_{f_{31}}\right\rangle_{M} .
\end{aligned}
$$

For moduli polynomial $f_{32}$, we select $R_{A}=R_{B}=x+1$ and perform similar computation. Finally, we can obtain a new formula. This formula also consists of 30 multiplication $m_{i}$ 's. Except for $m_{9}$ and $m_{11}$, all other $m_{i}$ 's are the same as those in [7]. Careful comparison shows that coefficient $c_{13}$ in [7] is a summation of $20 m_{i}$ 's, but every $c_{i}$ in the new formula is a summation of no more than $19 m_{i}$ 's. However, if we set $R_{A}=R_{B}=x^{2}$ for $f_{31}$ and $R_{A}=R_{B}=x$ for $f_{32}$, we will obtain another formula in which $c_{13}$ is a summation of $21 m_{i}$ 's.

Summarizing the method used in the above two examples, we can obtained an algorithm to derive more Karatsuba-like formulae in $G F(2)[x]$, namely,

1. For each moduli polynomial $f_{i}$, define $S_{i}=G F(2)[x] /\left(f_{i}\right)^{*}$;

2. For each pair $\left(R_{A}, R_{B}\right) \in S_{i} \times S_{i}$, derive a formula using the generalized CRT;

3. Save this formula if it is a new one.

\section{CONCLUSIONS}

We have generalized the division algorithm, and presented a method to obtain more $n$-term Karatsuba-like formulae in $G F(2)[x]$ for a fixed set of moduli polynomials. These new $n$-term formulae have the same multiplication complexity as that obtained from the conventional CRT. As for the addition complexity, we have checked some 4, 5, 6, 7, 8, and 9-term new formulae, but have not found obvious advantage or disadvantage. Even though, the proposed method can provide us with a broader understanding of Karatsuba-like formulae.

\section{ACKNOWLEDGMENT}

The work was supported by NSFC under grant No. 60970147. 


$$
\begin{aligned}
& m_{1}=\left(a_{0}+a_{1}+a_{2}+a_{4}+a_{3}+a_{5}+a_{6}+a_{7}+a_{8}\right)\left(b_{0}+b_{1}+b_{2}+b_{4}+b_{3}+b_{5}+b_{6}+b_{7}+b_{8}\right) ; \\
& m_{2}=\left(a_{0}+a_{2}+a_{4}+a_{6}+a_{8}\right)\left(b_{0}+b_{2}+b_{4}+b_{6}+b_{8}\right) \text {; } \\
& m_{3}=\left(a_{3}+a_{5}+a_{8}+a_{1}+a_{2}\right)\left(b_{1}+b_{5}+b_{8}+b_{2}+b_{3}\right) ; \\
& m_{4}=\left(a_{0}+a_{2}+a_{3}+a_{5}+a_{6}+a_{8}\right)\left(b_{0}+b_{2}+b_{3}+b_{5}+b_{6}+b_{8}\right) \text {; } \\
& m_{5}=\left(a_{0}+a_{3}+a_{6}+a_{1}+a_{4}+a_{7}\right)\left(b_{0}+b_{3}+b_{6}+b_{1}+b_{4}+b_{7}\right) ; \\
& m_{6}=\left(a_{0}+a_{3}+a_{4}+a_{5}+a_{7}\right)\left(b_{0}+b_{3}+b_{4}+b_{5}+b_{7}\right) \text {; } \\
& m_{7}=\left(a_{2}+a_{6}+a_{1}+a_{3}+a_{8}\right)\left(b_{2}+b_{6}+b_{1}+b_{3}+b_{8}\right) \text {; } \\
& m_{8}=\left(a_{2}+a_{4}+a_{5}+a_{6}\right)\left(b_{2}+b_{4}+b_{5}+b_{6}\right) \text {; } \\
& m_{9}=\left(a_{0}+a_{1}+a_{2}+a_{5}+a_{7}+a_{8}\right)\left(b_{0}+b_{1}+b_{2}+b_{5}+b_{7}+b_{8}\right) \text {; } \\
& m_{10}=\left(a_{1}+a_{3}+a_{5}+a_{7}\right)\left(b_{1}+b_{3}+b_{5}+b_{7}\right) ; \\
& m_{11}=\left(a_{0}+a_{1}+a_{2}+a_{4}+a_{7}+a_{8}\right)\left(b_{0}+b_{1}+b_{2}+b_{4}+b_{7}+b_{8}\right) ; \\
& m_{12}=\left(a_{7}+a_{0}+a_{3}+a_{5}+a_{6}\right)\left(b_{7}+b_{0}+b_{3}+b_{5}+b_{6}\right) ; \\
& m_{13}=\left(a_{0}+a_{1}+a_{4}+a_{5}+a_{8}\right)\left(b_{0}+b_{1}+b_{4}+b_{5}+b_{8}\right) ; \\
& m_{14}=\left(a_{1}+a_{2}+a_{4}+a_{5}+a_{7}+a_{8}\right)\left(b_{1}+b_{2}+b_{4}+b_{5}+b_{7}+b_{8}\right) ; \\
& m_{15}=\left(a_{0}+a_{1}+a_{3}+a_{6}+a_{7}+a_{8}\right)\left(b_{0}+b_{1}+b_{3}+b_{6}+b_{7}+b_{8}\right) ; \\
& m_{16}=\left(a_{1}+a_{3}+a_{4}+a_{5}+a_{8}\right)\left(b_{1}+b_{3}+b_{4}+b_{5}+b_{8}\right) ; \\
& m_{17}=\left(a_{0}+a_{2}+a_{3}+a_{4}+a_{7}\right)\left(b_{0}+b_{2}+b_{3}+b_{4}+b_{7}\right) \text {; } \\
& m_{18}=\left(a_{1}+a_{4}+a_{5}+a_{6}+a_{8}\right)\left(b_{1}+b_{4}+b_{5}+b_{6}+b_{8}\right) ; \\
& m_{19}=\left(a_{0}+a_{2}+a_{5}+a_{6}+a_{7}\right)\left(b_{0}+b_{2}+b_{5}+b_{6}+b_{7}\right) ; \\
& m_{20}=\left(a_{2}+a_{3}+a_{6}+a_{7}\right)\left(b_{2}+b_{3}+b_{6}+b_{7}\right) ; \\
& m_{21}=\left(a_{6}+a_{8}\right)\left(b_{6}+b_{8}\right) \text {; } \\
& m_{22}=\left(a_{0}+a_{2}\right)\left(b_{0}+b_{2}\right) \text {; } \\
& m_{23}=\left(a_{0}+a_{1}\right)\left(b_{0}+b_{1}\right) ; \\
& m_{24}=a_{0} b_{0} \\
& m_{25}=a_{1} b_{1} \text {; } \\
& m_{26}=a_{7} b_{7} \text {; } \\
& m_{27}=\left(a_{7}+a_{8}\right)\left(b_{7}+b_{8}\right) \text {; } \\
& m_{28}=a_{6} b_{6} \\
& m_{29}=a_{8} b_{8} ; \\
& m_{30}=a_{2} b_{2} \text {; }
\end{aligned}
$$




$$
\begin{aligned}
& c_{0}=m_{24} \\
& c_{1}=m_{24}+m_{25}+m_{23} \\
& c_{2}=m_{22}+m_{24}+m_{30}+m_{25} \\
& c_{3}=m_{22}+m_{30}+m_{23}+m_{13}+m_{20}+m_{10}+m_{14}+m_{4}+m_{16}+m_{7} \\
& +m_{8}+m_{12}+m_{18}+m_{6}+m_{3}+m_{21}+m_{28}+m_{29}+m_{27} \\
& c_{4}=m_{24}+m_{25}+m_{23}+m_{10}+m_{2}+m_{5}+m_{4}+m_{16}+m_{9}+m_{17} \\
& +m_{8}+m_{18}+m_{6}+m_{11}+m_{19}+m_{21}+m_{28}+m_{29}+m_{26} ; \\
& c_{5}=m_{22}+m_{24}+m_{30}+m_{25}+m_{1}+m_{10}+m_{2}+m_{14}+m_{4}+m_{16} \\
& +m_{17}+m_{12}+m_{6}+m_{15}+m_{19}+m_{29}+m_{26}+m_{27} \\
& c_{6}=m_{22}+m_{24}+m_{30}+m_{23}+m_{13}+m_{20}+m_{2}+m_{5}+m_{4}+m_{6} \\
& +m_{15}+m_{19}+m_{21}+m_{28}+m_{27} \\
& c_{7}=m_{24}+m_{1}+m_{16}+m_{7}+m_{8}+m_{12}+m_{6}+m_{15}+m_{19}+m_{21} \\
& +m_{28}+m_{29}+m_{26} \\
& c_{8}=m_{24}+m_{25}+m_{23}+m_{1}+m_{9}+m_{17}+m_{7}+m_{12}+m_{18}+m_{15} \\
& +m_{19}+m_{3}+m_{29}+m_{26}+m_{27} \\
& c_{9}=m_{22}+m_{24}+m_{30}+m_{25}+m_{1}+m_{16}+m_{9}+m_{7}+m_{18}+m_{15} \\
& +m_{11}+m_{29} \\
& c_{10}=m_{22}+m_{30}+m_{23}+m_{13}+m_{1}+m_{20}+m_{10}+m_{14}+m_{4}+m_{16} \\
& +m_{9}+m_{7}+m_{21}+m_{28}+m_{29}+m_{27} \\
& c_{11}=m_{24}+m_{25}+m_{23}+m_{1}+m_{10}+m_{2}+m_{5}+m_{4}+m_{16}+m_{9} \\
& +m_{7}+m_{18}+m_{6}+m_{3}+m_{21}+m_{28}+m_{29}+m_{26} \\
& c_{12}=m_{22}+m_{24}+m_{30}+m_{25}+m_{10}+m_{2}+m_{14}+m_{4}+m_{9}+m_{8} \\
& +m_{12}+m_{11}+m_{19}+m_{3}+m_{29}+m_{26}+m_{27} \\
& c_{13}=m_{22}+m_{24}+m_{30}+m_{23}+m_{13}+m_{1}+m_{20}+m_{2}+m_{5}+m_{4} \\
& +m_{16}+m_{17}+m_{12}+m_{18}+m_{15}+m_{11}+m_{21}+m_{28}+m_{27} \\
& c_{14}=m_{21}+m_{28}+m_{29}+m_{26} \\
& c_{15}=m_{29}+m_{26}+m_{27} \\
& c_{16}=m_{29} ;
\end{aligned}
$$




\section{REFERENCES}

[1] A. Karatsuba, "The complexity of computations," Proc. Steklov Inst. Math. vol. 211, pp.169-183, 1995.

[2] A. Karatsuba and Y. Ofman, "Multiplication of Multidigit Numbers on Automata," Soviet Physics-Doklady (English translation), vol. 7, no. 7, pp. 595-596, 1963.

[3] A. Lempel, G. Seroussi and S. Winograd, "On the Complexity of Multiplication in Finite Fields," Theoretical Computer Science, vol. 22, pp. 285-296, 1983

[4] A. Weimerskirch, and C. Paar, "Generalizations of the Karatsuba Algorithm for Efficient Implementations," http : //eprint.iacr.org/2006/224, 2003.

[5] P. L. Montgomery, "Five, Six, and Seven-Term Karatsuba-Like Formulae," IEEE Transactions on Computers, vol. 54, no. 3, pp. 362-369, Mar. 2005.

[6] H. Fan and M. A. Hasan, "Comments on "Five, Six, and Seven-Term Karatsuba-Like Formulae"," IEEE Transactions on Computers, vol. 56, no. 5, pp. 716-717, May 2007.

[7] M. Cenk and F. Özbudak, "Improved Polynomial Multiplication Formulas over $\mathbb{F}_{2}$ Using Chinese Remainder theorem," IEEE Transactions on Computers, vol. 58, no. 4, pp. 572-576, April 2009.

[8] I. Oseledets, "Optimal Karatsuba-like formulae for certain bilinear forms in GF(2)," Linear Algebra and its Applications, vol. 429 pp. 2052-2066, 2008.

[9] M. Cenk, Ç. K. Koç and F. Özbudak, "Polynomial Multiplication over Finite Fields using Field Extensions and Interpolation," Proc. 19th IEEE International Symposium on Computer Arithmetic, 2009.

[10] J. V. Z. Gathen and J. Gerhard, "Modern Computer Algebra," Cambridge Univ. Press, First ed., 1999, Second ed., 2003.

[11] P. L. Montgomery, "Modular multiplication without trial division," Mathematics of Computation, 44(170), pp. 519-521, 1985.

[12] T. M. Apostol, "Introduction to Analytic Number Theory," Springer, 1976. 